\title{
Avoiding 7-circuits in 2-factors of cubic graphs
}

\author{
Robert Lukoťka* \\ Department of Mathematics and Computer Science \\ Faculty of Education, Trnava University \\ Trnava, Slovakia \\ robert.lukotka@truni.sk
}

Submitted: May 16, 2014; Accepted: Oct 24, 2014; Published: Nov 6, 2014

Mathematics Subject Classifications: 05C70, 90C27

\begin{abstract}
Let $G$ be a cyclically 4-edge-connected cubic graph with girth at least 7 on $n$ vertices. We show that $G$ has a 2 -factor $F$ such that at least a linear amount of vertices is not in 7 -circuits of $F$. More precisely, there are at least $n / 657$ vertices of $G$ that are not in 7-circuits of $F$. If $G$ is cyclically 6 -edge-connected then the bound can be improved to $n / 189$. As a corollary we obtain bounds on the oddness and on the length of the shortest travelling salesman tour in a cyclically 4-edge-connected (6-edge-connected) cubic graph of girth at least 7 .
\end{abstract}

\section{Introduction}

According to the theorem of Vizing [8], each cubic graph has chromatic index 3 or 4 . A snark is a cubic graph with chromatic index 4 that is "non-trivial". Cubic graphs with bridges are considered trivial since they always have chromatic index 4 . However, in various contexts the requirements for being "non-trivial" may differ. Two most common measures of "non-triviality" are cyclic connectivity and girth. A graph is cyclically $k$ edge-connected if there is no edge cut-set of size less than $k$ separating two components both containing a circuit. The girth of a graph is the length of its shortest circuit.

In 1980 Jaeger and Swart [6] proposed a conjecture that every cyclically 7-edgeconnected cubic graph is 3-edge-colourable. The positive answer to this conjecture would significantly impact our knowledge on the structure of snarks. But since 1980 very little progress on this conjecture has been made. Even if we replace the 3-edge-colourability with the existence of a nowhere-zero 5-flow (cubic graph is 3-edge-colourable if and only if

*The work was partially supported by VEGA grant No. 1/0042/14. 
it has a nowhere-zero 4-flow [3, Proposition 6.4.5]) and replace 7 by any greater constant, then the problem is still unsolved. Therefore, if the conjecture is true, then it seems to be very difficult to prove.

If we colour edges of a cubic graph $G$ by 3 colours, then the union of the edges coloured by any two colours is a 2-factor containing only even circuits. On the other hand, if we have such a 2-factor we easily find a 3-edge-colouring. Hence the conjecture of Jaeger and Swart is equivalent to the statement "Every cyclically 7-edge-connected cubic graph $G$ has a 2-factor containing no odd circuits."

A natural weakening of this statement is to avoid only circuits of certain odd length. This leads to the following (much weaker) conjecture.

Conjecture 1. Each cyclically 7-edge-connected cubic graph has a 2-factor without 7circuits.

Nevertheless, this conjecture still seems to be very hard. To approach it we prove that at least a significant amount of vertices of a cyclically 7-edge-connected cubic graph $G$ is outside 7-circuits in some 2-factor of $G$.

Another motivation for this research arises from previous work on oddness [7]. The oddness of a bridgeless cubic graph $G$, denoted by $\omega(G)$, is the minimal number of odd circuits in a 2-factor of $G$. Apparently, a graph $G$ has oddness 0 if and only if it is 3edge-colourable. The following conjecture bounds how large the oddness of a snark might be.

Conjecture 2. [7] If $G$ is a bridgeless cubic graph, then $|V(G)| \geqslant 7.5 \omega(G)-5$.

The paper [2] makes a significant progress in avoiding 5-circuits in 2-factors of cubic graphs. However, to prove Conjecture 2 we also need to avoid some circuits of length 7 . The techniques from [7] and [2] cannot be applied to 7-circuits and cannot even guarantee the existence of a single circuit of length different from 7 in some 2 -factor. To prove Conjecture 2 we need to develop new tools that allow us to avoid 7-circuits in a 2-factor of a cubic graph.

Also, one of the reason why we decided to study circuits of length 7 is that avoiding them seems to be significantly more difficult than e.g. avoiding circuits of length 6 (we sketch one of the methods in the last section). Indeed, we were able to guarantee only small (but linear) fraction of vertices not being in circuits of length 7 . Moreover, we require the graph to be cyclically 4 -edge-connected and have girth at least 7 . This is the main result of the paper.

Theorem 3. Let $G$ be a cubic graph of girth at least 7 on $n$ vertices.

- If $G$ is cyclically 4-edge-connected, then there is a 2-factor $F$ of $G$ such that at least $n / 657$ vertices of $G$ are not in 7 -circuits of $F$.

- If $G$ is cyclically 6-edge-connected, then there is a 2-factor $F$ of $G$ such that at least $n / 189$ vertices of $G$ are not in 7 -circuits of $F$. 
The result implies a first non-trivial bound on oddness ratio $(|V(G)| / \omega(G))$ of cyclically 7-edge-connected snarks (see [7] for more information on oddness ratio). As another corollary we bound the length of the shortest travelling salesman tour, which is a closed walk containing all vertices of the graph, for cyclically 4-edge-connected (6-edge-connected) graphs of girth at least 7 . Although, the improvements are small, they present the first non-trivial bounds for investigated classes of graphs. The proofs of the results in this paper are constructive and yield polynomial time algorithms.

\section{Avoiding 7-circuits in snarks of girth 7}

Proof of Theorem 3. The proof of both statements follows the same arguments. We present the proof of the statement assuming cyclic 4-edge-connectivity. The modifications required when the cyclic connectivity is increased to 6 will be given in brackets (mostly, only the values are different).

We fix an arbitrary 2-factor $F$ of $G$. This 2-factor will be fixed throughout the proof of the theorem. The circuits of $F$ will be referred to as $F$-circuits to distinguish them from other circuits. Each vertex $v$ of $G$ is in exactly one $F$-circuit. We will denote this circuit by $F(v)$. We say that two $F$-circuits are neighbours if an edge connects them. The set of $F$-circuits that are neighbours with a $F$-circuit $C$ will be denoted by $N_{F}(C)$. The set of vertices outside $C$ that are neighbours to some vertex from $C$ will be denoted by $N_{V}(C)$. We say that two $F$-circuits are $k$-neighbours if exactly $k$ edges connect them.

Let $\mathcal{C}_{8+}$ be the set of circuits from $F$ of length more than 7 . Let $\mathcal{N}_{8+}$ be the set of circuits from $F$ that are neighbours with some circuit from $\mathcal{C}_{8+}$. Let $m$ be the number of vertices in the circuits from $\mathcal{C}_{8+}$. If $m \geqslant n / 657$ [or $m \geqslant n / 189$ in case $G$ is 6 -edgeconnected], then the theorem is true. Therefore we can assume

$$
m<n / 657[<n / 189] \text {. }
$$

There are $(n-m) / 7$ circuits of length 7 in $F$.

We create an auxiliary graph $G_{B}$ as follows: we contract each circuit of $F$, we delete vertices created by contracting circuits from $\mathcal{C}_{8+}$ and $\mathcal{N}_{8+}$. Each vertex in $G_{B}$ has degree at most 7 . We square $G_{B}$ (that is we add an edge between each pair of vertices in distance 2 ) and denote the new graph $G_{A}$. Maximal degree in $G_{A}$ is at most 49. Because the number of circuits in $\mathcal{N}_{8+}$ is at most $m$ we know that graph $G_{A}$ has at least $(n-m) / 7-m=$ $(n-8 m) / 7$ vertices.

Suppose first that $G_{A}$ is not isomorphic to $K_{50}$. By Brooks' theorem $G_{A}$ is 49-vertexcolourable. Therefore $G_{A}$ has an independent set $S$ of size at least $(n-8 m) / 343$. Suppose on the other hand $G_{A}$ is isomorphic to $K_{50}$. Then $m=0$ and $G_{A}$ has an independent set $S$ of size $n / 350$. In both cases $|S| \geqslant \min \{n / 350,(n-8 m) / 343\}$. The $F$-circuits that correspond to vertices from $S$ will be called chosen circuits. The set of chosen circuits will be denoted by $\mathcal{C}$.

A chosen circuit $C \in \mathcal{C}$ is a neighbour of several other $F$-circuits. The following observation describes some of the properties of the chosen circuits of $G$. 
Observation 4. The following statements hold:

A. Each chosen circuit $C$ is a 7-circuit. All F-circuits that are neighbours with $C$ are 7-circuits.

B. No two chosen circuits are neighbours. No F-circuit is neighbour with two chosen circuits.

C. Chosen circuits have no chord.

D. If a chosen circuit $C$ has a $k$-neighbour $C^{\prime}, k \geqslant 2$, then the edges connecting $C$ and $C^{\prime}$ are not incident to consecutive vertices in both $C$ and $C^{\prime}$.

E. A circuit $C \in \mathcal{C}$ has no $k$-neighbour for $k>2$.

Proof. We prove each statement separately.

$A$. While constructing $G_{A}$ we deleted vertices corresponding to circuits from $\mathcal{C}_{8+}$ and $\mathcal{N}_{8+}$. This implies the statement.

$B$. The two chosen circuits that are neighbours to a single $F$-circuit correspond to vertices in distance 2 in $G_{B}$ and thus they are neighbours in $G_{A}$. This contradicts the choice of $S$ and thus the choice of $\mathcal{C}$. Similarly, no two chosen circuits can be neighbours.

$C$. By statement A each chosen circuit $C$ is a 7 -circuit. If $C$ had chord, then there would be circuit of length less than 7 , contradicting the girth 7 condition.

$D$. By statement $\mathrm{A}$ both $C$ and $C^{\prime}$ are 7-circuits. Let $C=v v_{1} v_{2} \ldots v_{6}$ and $C^{\prime}=$ $v^{\prime} v_{1}^{\prime} v_{2}^{\prime} \ldots v_{6}^{\prime}$. Suppose for the contrary, that there are two edges contradicting the statement. Without loss of generality suppose that these edges are $v v^{\prime}$ and $v_{1} v_{i}^{\prime}$ for some $i \in\{1,2,3\}$. It can be easily verified that a circuit of length less than 7 exists, contradicting the girth 7 condition.

$E$. For the contrary, let $C^{\prime}$ be a $k$-neighbour of $C$, where $k>2$. By statement A both $C$ and $C^{\prime}$ are 7 -circuits. Let $C=v v_{1} v_{2} \ldots v_{6}$ and $C^{\prime}=v^{\prime} v_{1}^{\prime} v_{2}^{\prime} \ldots v_{6}^{\prime}$. We may without loss of generality (using statement D) assume that the edges connecting $C$ and $C^{\prime}$ have endvertices $v, v_{2}, v_{5}, v^{\prime}, v_{2}^{\prime}$, and $v_{5}^{\prime}$. Moreover we may without loss of generality assume that $v_{5} v_{5}^{\prime}$ is one of the connecting edges. But then circuit containing the vertex-set $\left\{v, v_{1}, v_{2}, v^{\prime}, v_{1}^{\prime}, v_{2}^{\prime}\right\}$ has length 6 - a contradiction.

For each $C \in \mathcal{C}$ we construct a quadruple $\left(v, e_{1}, e_{2}, C\right)$ as follows. By Observation $4 \mathrm{~A}$ circuit $C$ is a 7 -circuit and by Observation $4 \mathrm{E}$ circuit $C$ must have a 1 -neighbour $C^{\prime}$. We pick an arbitrary circuit $C^{\prime}$ with this property and we set $v$ to be the unique vertex from $C \cap N_{V}\left(C^{\prime}\right)$. We denote the other vertices of $C$ as $v_{1}, v_{2}, \ldots, v_{6}$ in consecutive order (so that $v$ is neighbour of $v_{1}$ and $v_{6}$ ). Out of the two choices how to do this we choose an arbitrary one. We denote the neighbours of $v, v_{1}, v_{2}, \ldots, v_{6}$ from $N_{V}(C)$ as $w, w_{1}, w_{2}, \ldots$, $w_{6}$, respectively. We set $e_{1}=v v_{6}$ and $e_{2}=v_{2} w_{2}$. We create a quadruple for each $C \in \mathcal{C}$ 
and denote the resulting collection of quadruples as $\mathcal{T}$. The number of such quadruples is equal to $|\mathcal{C}|$, that is

$$
|\mathcal{T}| \geqslant \min \{n / 350,(n-8 m) / 343\} .
$$

Let $\left(v, e_{1}, e_{2}, C\right) \in \mathcal{T}$. The position of $e_{1}$ and $e_{2}$ with respect to $v$ is depicted in Figure 1 - the bold edges are $e_{1}$ and $e_{2}$. Let $M_{1}$ be some 1-factor of $G$. If $e_{1}, e_{2} \in M_{1}$, then we say that $M_{1}$ fulfils the quadruple $\left(v, e_{1}, e_{2}, C\right)$. Let $F_{1}$ be the 2 -factor complementary to $M_{1}$.

Observation 5. If $M_{1}$ fulfils $\left(v, e_{1}, e_{2}, C\right)$, then circuit $C^{*}$ of $F_{1}$ containing $v$ has at least 8 vertices from $C \cup N_{F}(C)$.

Proof. As $e_{1}, e_{2} \in M_{1}$ the circuit $C^{*}$ contains edges $w v, v v_{1}, v_{1} v_{2}, v_{2} v_{3}$. Because $e_{1}=$ $v v_{6} \in M_{1}$ we have $C \neq C^{*}$. Therefore there is a vertex $w_{i} \in C^{*}$, for some $i \in\{3,4,5,6\}$. Then $C^{*}$ contains at least $w, v, v_{1}, v_{2}, v_{3}, w_{i}$, at least one neighbour of $w$ from $F(w)$, and at least one neighbour of $w_{i}$ from $F\left(w_{i}\right)$ (note that $F(w) \neq F\left(w_{i}\right)$, due to choice of $v$ ). So the statement follows.
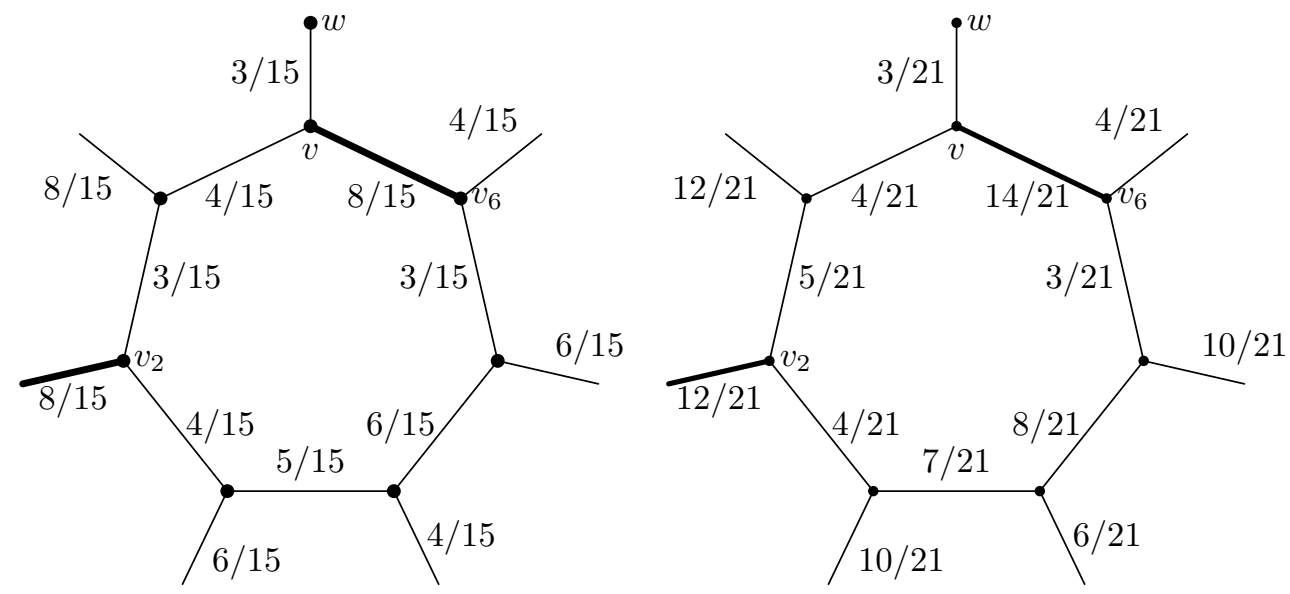

Figure 1: Modification of $\mathbf{x}_{0}$ on $C$ and on the boundary of $C$.

Our aim is to find a perfect matching that fulfils as many quadruples from $\mathcal{T}$ as possible. Let $\mathbf{x}$ be a weighting of edges with non-negative real numbers. A weighting of the edges of $G$ is sum-correct on a vertex if the sum of weights on edges incident with that vertex is 1 . A perfect matching can be viewed as a sum-correct weighting of edges using only weights 0 and 1 . The perfect matching polytope [4] of $G$, denoted by $\mathcal{P}(G)$, is the convex hull of all perfect matchings on $G$. Suppose $\mathbf{x}$ is a point of $\mathcal{P}(G)$. The symbol $x_{e}$ will denote the coordinate of $\mathbf{x}$ corresponding to edge $e$. We define a linear function

$$
f_{\mathcal{T}}(\mathbf{x})=\sum_{\left(v, e_{1}, e_{2}, C\right) \in \mathcal{T}}\left(x_{e_{1}}+x_{e_{2}}\right) .
$$


We optimize the value of $f_{\mathcal{T}}$ in $\mathcal{P}(G)$. Since $f_{\mathcal{T}}$ is linear the maximum of $f_{\mathcal{T}}$ is attained in a vertex of $\mathcal{P}(G)$, thus some optimal fractional perfect matching is a perfect matching of $G$. In the next few paragraphs we find a point $\mathbf{x}^{*}$ within the perfect matching polytope, such that $f_{\mathcal{T}}\left(\mathbf{x}^{*}\right)=(1+1 / 15)|\mathcal{T}|[=(1+5 / 21)|\mathcal{T}|]$. This guarantees the existence of a perfect matching (non-fractional) $\mathbf{x}^{\mathrm{OPT}}$ such that $f_{\mathcal{T}}\left(\mathrm{x}^{\mathrm{OPT}}\right) \geqslant(1+1 / 15)|\mathcal{T}|[\geqslant(1+5 / 21)|\mathcal{T}|]$.

We assign all edges of $G$ the initial weights $\mathbf{x}_{0}=(1 / 3,1 / 3, \ldots, 1 / 3)$. A weighting of the edges of $G$ is central if for each edge $e$ we have $1 / 5 \leqslant x_{e} \leqslant 3 / 5$ [or $1 / 7 \leqslant x_{e} \leqslant 5 / 7$. Clearly $\mathbf{x}_{0}$ is central and sum-correct on all vertices of $G$. For each quadruple $\left(v, e_{1}, e_{2}, C\right) \in \mathcal{T}$ we modify $\mathbf{x}_{0}$ on $C$, on the boundary of $C$, which is the set of edges with exactly one end in $C$, and on circuits from $N_{F}(C)$. By Observation $4 \mathrm{~B}$ the modifications for distinct quadruples will not interfere.

For each quadruple $\left(v, e_{1}, e_{2}, C\right) \in \mathcal{T}$ we do the following modifications. By Observation $4 \mathrm{~A}$ and Observation $4 \mathrm{C}$ the circuit $C$ is a 7 -circuit and has no chord. First, we modify the values of $\mathbf{x}_{0}$ on $C$ and on the boundary of $C$ as depicted on Figure 1 left [right]. The resulting weighting central is sum-correct on all vertices except for vertices from $N_{V}(C)$. Let $C_{N} \in N_{F}(C)$. By Observation $4 \mathrm{~A}$ circuit $C_{N}$ is an 7-circuit. By Observation $4 \mathrm{E}$ it is either 1-neighbour of $C$ or a 2-neighbour of $C$. Denote the vertices of $C_{N}$ as $v_{N}, v_{N 1}, v_{N 2}$, $\ldots, v_{N 6}$ in consecutive order so that $v_{N} \in N_{V}(C)$, and if $C_{N}$ is a 2-neighbour of $C$, either $v_{N 4} \in N_{V}(C)$ or $v_{N 5} \in N_{V}(C)$ (this is guaranteed by Observation 4D). Let $a$ and $b$ (if $C_{N}$ is a 2-neighbour of $C$ ) denote the weights of edges connecting $C$ and $C_{N}$ (as in Figure 2 ). We replace the current weights with weights from Figure 2. The resulting weighting is sum-correct on vertices from $C_{N}$. Moreover, we can easily check that the weighting is central provided $3 / 15 \leqslant a, b \leqslant 9 / 15,|a-b| \leqslant 4 / 15$, and $a+b \leqslant 14 / 15$ [provided $3 / 21 \leqslant a, b \leqslant 15 / 21,|a-b| \leqslant 8 / 21$, and $a+b \leqslant 22 / 21]$. As $F(w)$ is a 1-neighbour of $C$ by choice of $v$ and using Observation 4D, these condition are always satisfied (see Figure 1). After we perform the modifications for each $C_{N} \in N_{F}(C)$ we get a weighting that is central and sum-correct on all vertices from $C \cup N_{F}(C)$.

We do the weighting modifications for each quadruple from $\mathcal{T}$. We denote the resulting weighting by $\mathbf{x}^{*}$. From the construction of $\mathbf{x}^{*}$ we have $f_{\mathcal{T}}\left(\mathbf{x}^{*}\right)=16 / 15 \cdot|\mathcal{T}|[=26 / 21 \cdot|\mathcal{T}|]$. We show that $\mathrm{x}^{*} \in \mathcal{P}(G)$. Due to characterisation by Edmonds [4] the perfect matching polytope can be described by three sets of equations:

1. Edges have non-negative weights.

2. For each vertex the sum of weights on incident edges is 1 .

3. For each edge-cut separating a subgraph $U$ with an odd number of vertices the sum of the weights on the boundary of $U$ is at least 1 .

First two properties are guaranteed by the fact that $\mathrm{x}^{*}$ uses non-negative weights and is sum-correct on all vertices. We need only to check the third property. Due to parity constraints the edge cuts separating subgraph with odd number of vertices must be odd. As $G$ is cyclically 4-edge-connected each 3-edge-cut in $G$ separates only one vertex, we denote it $v_{U 1}$. As $\mathbf{x}^{*}$ is sum-correct on $v_{U 1}$ the property 3 is satisfied for such a cut. Larger cuts satisfy the property 3 because $\mathbf{x}^{*}$ is central. 


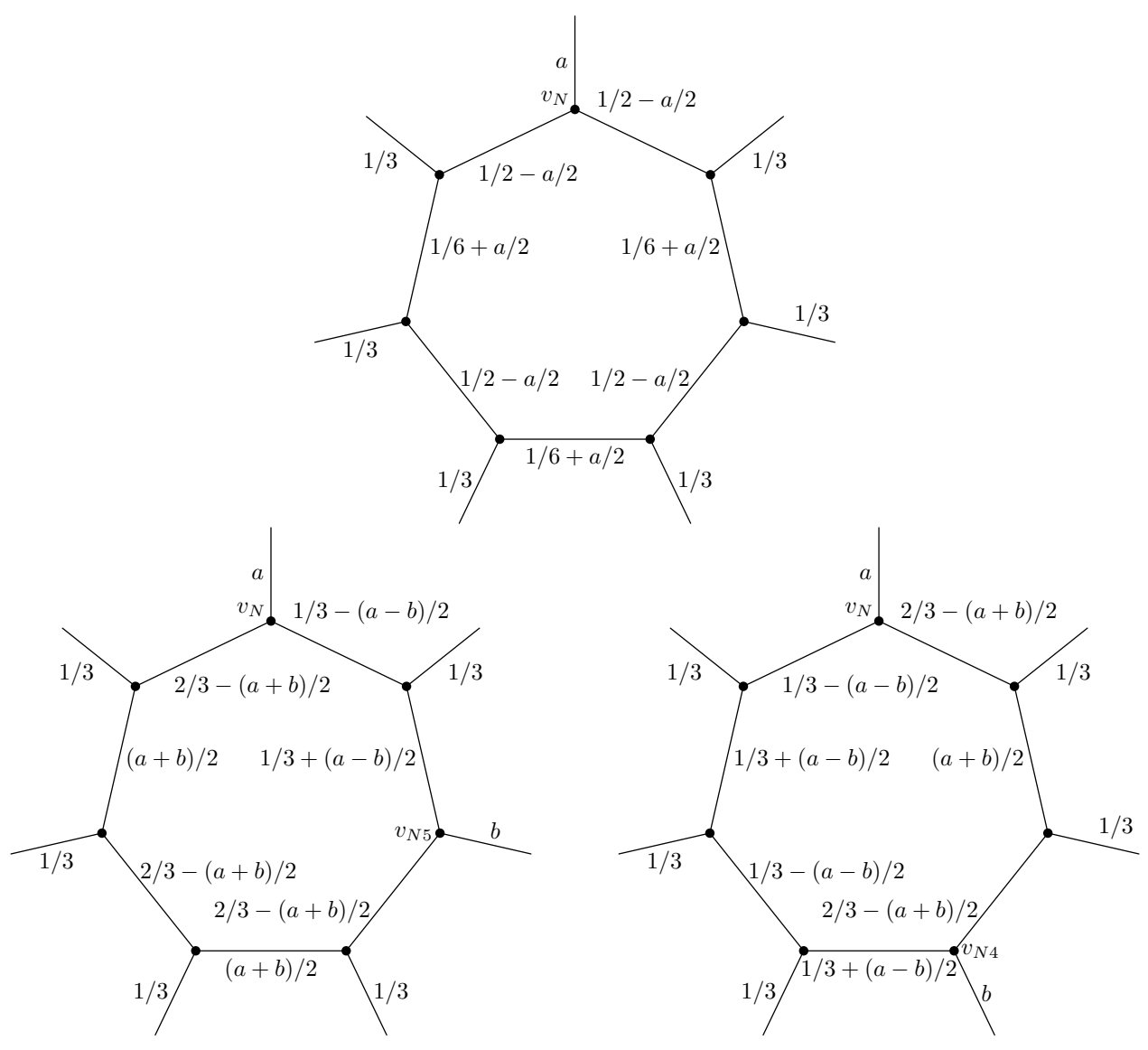

Figure 2: Weights of $C_{N}$.

[If $G$ is cyclically 6-edge-connected, then only trivial (separating an acyclic subgraph) 5-cuts exist. Such a cut separates a 2-path. Let us denote the vertices of such a path as $v_{U 1}, v_{U 2}, v_{U 3}$. The weights of cut-edges incident to $v_{U 1}$ will be denoted by $u_{1}$ and $u_{2}$. The weight of an cut-edge incident to $v_{U 2}$ will be denoted by $u_{3}$. The weights of cut-edges incident to $v_{U 3}$ will be denoted by $u_{4}$ and $u_{5}$. As $\mathbf{x}^{*}$ is sum-correct we have $u_{1}+u_{2}+x_{v_{U 1} v_{U 2}}=1, x_{v_{U 1} v_{U 2}}+u_{3}+x_{v_{U 2} v_{U 3}}=1$, and $x_{v_{U 2} v_{U 3}}+u_{4}+u_{5}=1$. We can combine these equations into $u_{1}+u_{2}-u_{3}+u_{4}+u_{5}=1$. Therefore $u_{1}+u_{2}+u_{3}+u_{4}+u_{5} \geqslant 1$. Larger cuts satisfy the property 3 because $\mathbf{x}^{*}$ is central.]

We have found $\mathbf{x}^{*} \in \mathcal{P}(G)$ such that $f_{\mathcal{T}}\left(\mathbf{x}^{*}\right) \geqslant(1+1 / 15)|\mathcal{T}| \quad[$ or $\geqslant(1+5 / 21)|\mathcal{T}|]$. Therefore there exist a 1 -factor $\mathbf{x}^{\mathrm{OPT}}$ such that $f_{\mathcal{T}}\left(\mathbf{x}^{\mathrm{OPT}}\right) \geqslant(1+1 / 15)|\mathcal{T}| \quad$ or $\geqslant(1+$ $5 / 21)|\mathcal{T}|$. Since $\mathbf{x}^{\mathrm{OPT}}$ is a perfect matching each quadruple in $\mathcal{T}$ adds 0 , 1 , or 2 to the sum (3). Therefore at least $1 / 15|\mathcal{T}|$ [or $5 / 21|\mathcal{T}|]$ of the quadruples from $\mathcal{T}$ add 2 to the sum (3). Perfect matching $\mathbf{x}^{\mathrm{OPT}}$ fulfils all these quadruples. Thus $\mathbf{x}^{\mathrm{OPT}}$ fulfils at least $1 / 15|\mathcal{T}|$ [at least $5 / 21|\mathcal{T}|]$ quadruples from $\mathcal{T}$.

Moreover, by Observation 5 each quadruple $\left(v, e_{1}, e_{2}, C\right)$ that is fulfilled by $\mathbf{x}^{\mathrm{OPT}}$ yields at least 8 vertices in $C$ and $N_{F}(C)$ that are outside 7 -circuits of the 2-factor complementary to $\mathrm{x}^{\mathrm{OPT}}$. By Observation $4 \mathrm{~B}$ the set of 8 vertices is disjoint for different quadruples. 
Therefore the number of vertices of $G$ that are not in 7-circuit of 2-factor complementary to $\mathbf{x}^{\mathrm{OPT}}$ is at least $8 \cdot \frac{1}{15}|\mathcal{T}|\left[\right.$ or $\left.8 \cdot \frac{5}{21}|\mathcal{T}|\right]$. This number can be bounded using (1) and (2):

$$
\begin{gathered}
8 \cdot \frac{1}{15}|\mathcal{T}| \geqslant \frac{8}{15} \cdot \min \left\{\frac{n}{350}, \frac{n-8 m}{343}\right\} \geqslant \frac{8}{5250} n \geqslant \frac{n}{657} . \\
{\left[8 \cdot \frac{5}{21}|\mathcal{T}| \geqslant \frac{40}{21} \cdot \min \left\{\frac{n}{350}, \frac{n-8 m}{343}\right\} \geqslant \frac{40-\frac{320}{189}}{7203} n \geqslant \frac{n}{189} .\right]}
\end{gathered}
$$

We proceed with two corollaries of Theorem 3. We can bound the oddness as well as the length of the shortest travelling salesman tour of cubic graph of girth at least 7 and cyclic connectivity at least 4 .

Corollary 6. Let $G$ be a snark of girth at least 7 on $n$ vertices.

- If $G$ is cyclically 4-edge-connected, then $\omega(G)<\frac{n}{7.0024}$.

- If $G$ is cyclically 6-edge-connected, then $\omega(G)<\frac{n}{7.0082}$.

Proof. We choose a 2-factor according to Theorem 3. If $G$ is cyclically 4-edge-connected, then we have at most $c_{7} \leqslant 656 n /(7 \cdot 657)$ circuits of length 7 . The remaining vertices can form at most $\left(n-7 c_{7}\right) / 9$ odd circuits. Total number of odd circuits is therefore at most

$$
c_{7}+\frac{n-7 c_{7}}{9}=\frac{n+2 \cdot c_{7}}{9} \leqslant\left[\frac{1+\frac{1312}{7 \cdot 657}}{9}\right] n<\frac{n}{7.0024} .
$$

For $G$ cyclically 6-edge-connected the maximal number of odd circuits is

$$
c_{7}+\frac{n-7 c_{7}}{9}=\frac{n+2 \cdot c_{7}}{9} \leqslant\left[\frac{1+\frac{376}{7 \cdot 189}}{9}\right] n<\frac{n}{7.0082} .
$$

Corollary 7. Let $G$ be a cubic graph of girth at least 7 on $n$ vertices.

- If $G$ is cyclically 4-edge-connected, then $G$ has a travelling salesman tour of length less than $1.28566 \cdot n-2$.

- If $G$ is cyclically 6-edge-connected, then $G$ has a travelling salesman tour of length less than $1.28553 \cdot n-2$.

Proof. Let $n$ denote the number of vertices of $G$. We choose a 2-factor $F$ according to Theorem 3. If $G$ is cyclically 4 -edge-connected, then we have at most $c_{7} \leqslant 656 n /(7 \cdot 657)$ circuits of length 7 . The remaining vertices can form at most $\left(n-7 c_{7}\right) / 8$ circuits. We contract the circuits of $F$ and chose a spanning tree $T$ in the resulting graph. This 
spanning tree contains at most $c_{7}+\left(n-7 c_{7}\right) / 8-1$ edges. We use edges from $T$ twice and edges from $F$ once. Altogether the length of the tour is at most

$$
n+2\left(c_{7}+\frac{n-7 c_{7}}{8}-1\right)=\frac{5}{4} n+\frac{1}{4} c_{7}-2 \leqslant 5 / 4 n+\frac{656}{28 \cdot 657} n-2<1.28566 n-2 .
$$

For $G$ cyclically 6-edge-connected the bound improves to $1.28553 n-2$.

This is a slight improvement compared to trivial bound of $9 n / 7-2 \approx 1.28572 n-2$.

\section{Remarks}

The proof of Theorem 3 can be slightly modified in such a way that instead of optimisation argument we use a probabilistic one. Indeed, in a key part of the proof of Theorem 3 we construct a point $\mathbf{x}^{*}$ within the perfect matching polytope such that $f_{\mathcal{T}}\left(\mathbf{x}^{*}\right)$ is high enough. The fact that $\mathbf{x}^{*} \in \mathcal{P}(G)$ implies it is a convex combination of some perfect matchings $\mathbf{x}_{1}, \mathbf{x}_{2}, \ldots \mathbf{x}_{l}$.

$$
\mathbf{x}^{*}=\alpha_{1} \mathbf{x}_{1}+\alpha_{2} \mathbf{x}_{2}+\cdots+\alpha_{l} \mathbf{x}_{l} .
$$

We choose a random perfect matching $\mathbf{x}_{R}$ as follows: perfect matching $\mathbf{x}_{i}$ is chosen with probability $\alpha_{i}$, for $i \in\{1,2, \ldots, l\}$. For the linear function $f_{\mathcal{T}}$ the expected value $E\left(f_{\mathcal{T}}\left(\mathbf{x}_{R}\right)\right)=f_{\mathcal{T}}\left(\mathbf{x}^{*}\right)$. This implies that for some perfect matching $\mathbf{x}_{j}, j \in\{1,2, \ldots, l\}$, we have $f_{\mathcal{T}}\left(\mathbf{x}_{j}\right) \geqslant f_{\mathcal{T}}\left(\mathbf{x}^{*}\right)$. If one could find non-trivial bounds on correlation, e.g. between events $\left(v, e_{1}, e_{2}, C\right)$ is fulfilled and $\left(v^{\prime}, e_{1}^{\prime}, e_{2}^{\prime}, C^{\prime}\right)$ is fulfilled when $C$ and $C^{\prime}$ are distant (in some conveniently chosen probability space), results from the paper might improve significantly.

One of the drawbacks of our results is that we strongly rely on the fact that graph has girth at least 7 . Similar result without the girth 7 requirement would be appreciated. If girth 7 is substituted with girth 6 , then we would get a new bound on the oddness ratio of cyclically 6-edge-connected snarks (see [7] for details). To use the technique to approach Conjecture 2 the girth requirement needs to be decreased even more.

The approach taken in [7] and [2] for 5-circuits is not viable for 7-circuits. Similarly, our approach does not work for other circuit lengths. A natural goal is to generalize our results to graphs of higher girth of course with longer circuits to avoid. Although, the cyclic connectivity assumption might be omitted from the problem, we state the problem with this assumption as we believe it makes the problem more tractable.

Problem 8. Is it true that every cyclically 4-edge-connected cubic graph (could be replaced with higher cyclic connectivity requirement) of girth $k \geqslant 4$ has a 2 -factor such that a linear amount of vertices is not in circuits of length $k$ ?

If we exclude Petersen graph, then the problem has affirmative answer for all $k \in$ $\{4,5,6,7\}$. For $k=4$ the proof is relatively straightforward.

Theorem 9. Let $G$ be a bridgeless simple cubic graph on $n$ vertices, where $n>4$. Let $\mathcal{C}_{4}^{I}$ denote the set of circuits of $G$ of length 4 that do not intersect with other circuits of length 4 . Then $G$ has a 2-factor containing at most $\left|\mathcal{C}_{4}^{I}\right| / 3$ circuits of length 4. 
Proof. For each circuit $C \in \mathcal{C}_{4}^{I}$ we pick an arbitrary edge from the boundary of $C$ and denote it $e_{C}$. We find a perfect matching $M$ such that the sum of the weights (a perfect matching can be viewed as a sum-correct weighting of the edges using only weights 0 and 1$)$ of the edges $e_{C}$ over all circuits in $\mathcal{C}_{4}^{I}$ is minimal. As the point $(1 / 3,1 / 3, \ldots, 1 / 3)$ is in $\mathcal{P}(G)$, for the optimal perfect matching the sum is at most $\left|\mathcal{C}_{4}^{I}\right| / 3$. Therefore, the optimal perfect matching has at most $\left|\mathcal{C}_{4}^{I}\right| / 3$ circuits of length 4 from $\mathcal{C}_{4}^{I}$ in the in the complementary 2-factor $F$.

Suppose that $F$ contains a circuit $C$ of length 4 not in $\mathcal{C}_{4}^{I}$. Then $C$ is intersecting a circuit $C^{\prime}$ of length 4 . As $C$ is a 2 -factor circuit, $C$ and $C^{\prime}$ intersect in exactly 2-vertices. Then we can swap which edges of $C^{\prime}$ are in $F$ to merge $C$ with some other circuit of $F$. If we do this for all circuits of $F$ of length 4 not in $\mathcal{C}_{4}^{I}$, then we get the desired 2-factor.

For $k=5$ the problem is settled in [7] and the constant is improved in [2]. We answered the problem for $k=7$ in the previous section. The following theorem answers the problem for $k=6$. As the aim of this theorem is to show that there is a 2-factor such that a linear amount of vertices is not in circuits of length 6 we did not focus on improving the constant. The proof is partially inspired by the proof of Theorem 1 in [1].

Theorem 10. A bridgeless cubic graph graph $G$ on $n$ vertices of girth at least 6 has a 2 -factor containing at most $11 n / 70$ circuits of length 6 .

Proof. Let $\mathcal{C}_{6}$ be the set of all circuits of length 6 in $G$. As $\mathbf{x}_{0}=(1 / 3,1 / 3, \ldots, 1 / 3)$ is in $\mathcal{P}(G)$ we can find a perfect matching $M_{2}$ and the corresponding weighting of edges $\mathbf{x}^{\prime}$ such that

$$
\sum_{e_{1} e_{2} e_{3} e_{4} e_{5} e_{6} \in \mathcal{C}_{6}} x_{e_{1}}^{\prime}+x_{e_{2}}^{\prime}+x_{e_{3}}^{\prime}+x_{e_{4}}^{\prime}+x_{e_{5}}^{\prime}+x_{e_{6}}^{\prime} \geqslant 2\left|\mathcal{C}_{6}\right|
$$

Let $F_{2}$ be the 2-factor complementary to $M_{2}$. If $F_{2}$ contains less than $11 n / 70$ circuits of length 6 , then the theorem is proved. If $F_{2}$ contains at least $11 n / 70$ circuits of length 6 then by (4) there exist at least $22 n / 70$ circuits of length 6 such that exactly three edges belong to $M_{2}$. We denote the set of these circuits $\mathcal{C}_{M}$. If two circuits from $\mathcal{C}_{M}$ intersect, then they must intersect also in an edge from $M_{2}$. Moreover, each edge can be in at most 8 circuits of length 6 : suppose the circuit contains given edge, there are at most 8 possibilities how to add next three edges to the circuit, by the girth constraint there is at most one possibility for the remaining two edges. Therefore, each circuit from $\mathcal{C}_{M}$ can intersect with at most 21 other circuits from $\mathcal{C}_{M}$. By Brooks' theorem there is a set of at least $n / 70$ mutually disjoint circuits from $\mathcal{C}_{M}$. Let $\mathcal{C}_{I}$ be one such set of mutually disjoint circuits.

We modify $M_{2}$ as follows. Let $C=v_{1} v_{2} v_{3} v_{4} v_{5} v_{6}$ be a circuit from $\mathcal{C}_{I}$. Without loss of generality suppose that the edges $v_{1} v_{2}, v_{3} v_{4}$, and $v_{5} v_{6}$ are in $M_{2}$ and the edges $v_{2} v_{3}, v_{4} v_{5}$, and $v_{6} v_{1}$ are in $F_{2}$. If at least two edges, say $v_{2} v_{3}$ and $v_{4} v_{5}$, are in 6 -circuits of $F_{2}$, then by the girth condition the edges $v_{2} v_{3}, v_{4} v_{5}$, and $v_{6} v_{1}$ belong to three different circuits $C_{1}, C_{2}$, and $C_{3}$ of $F_{2}$. If this is the case, then we exchange the edges $v_{1} v_{2}, v_{3} v_{4}$, and $v_{5} v_{6}$ by $v_{2} v_{3}$, $v_{4} v_{5}$, and $v_{6} v_{1}$ in $M_{2}$. This merges $C_{1}, C_{2}$, and $C_{3}$ into a single circuit $C^{*}$. We continue 
modifications until no $C \in \mathcal{C}_{I}$ contains two edges that are in a circuit of length 6 in $F_{2}$. This process is finite because the number of 6 -circuits decreases. We denote the resulting matching by $M_{3}$ and the resulting 2-factor by $F_{3}$

Now each $C \in \mathcal{C}_{I}$ contains at least 4 vertices that are not in 6 -circuits of $F_{3}$. Altogether, there are at least $4 n / 70$ vertices are outside 6 -circuits in $F_{3}$. That 2 -factor contains at most $11 n / 70$ circuits of length 6 .

So Problem 8 has affirmative answer for $k \leqslant 7$. For other values of $k$ the problem is open. We find it very likely that the problem has affirmative answer for all other values of $k$.

The highest known value of the cyclic connectivity of a non-hamiltonian cubic graph is 7. A natural question is whether there is an cyclically 8-edge-connected non-hamiltonian cubic graph. Hamiltonian circuit itself is a 2 -factor of $G$. It would be interesting to know what is the biggest length of a circuit (as a the function of the number of vertices) we can guarantee in a 2-factor of a graph with given cyclic connectivity. It seems to be hard to guarantee even the existence of circuits of length more than 8 in the 2-factor-only recenly Küngden and Richter [5] showed that a circuit of length at least 7 is guaranteed provided the graph is not isomorphic to Petersen graph. Therefore we propose the following problem:

Problem 11. For which $k>1$ there is an unbounded non-decreasing function $f$ such that each cyclically $k$-edge-connected cubic graph on $n$ vertices has a 2 -factor containing a circuit of length at least $f(n)$ ?

For $k \leqslant 3$ the answer to the problem is negative [5]. For other values of $k$ the problem is open. If there is no 8-edge-connected non-hamiltonian cubic graph, then for $k \geqslant 8$ we have even $f(n)=n$.

\section{References}

[1] S. Boyd, R. Sitters, S. van der Ster, L. Stougie. The traveling salesman problem on cubic and subcubic graphs. Math. Program. Ser. A, 144(1-2):227-245, 2014.

[2] B. Candráková, R. Lukotka. Avoiding 5-circuits in a 2-factor of cubic graphs. arXiv:1311.0512 [math.CO].

[3] R. Diestel. Graph Theory. Springer-Verlag, Heidelberg, 4th edition, Graduate Texts in Mathematics, Volume 173, ISBN 978-3-642-14278-9.

[4] J. Edmonds. Maximum matching and a polyhedron with $(0,1)$ vertices. J. Res. Nat. Bur. Standards Sect B. 69B(1-2):125-130, 1965.

[5] A. Kündgen, R. B. Richter. On 2-factors with long cycles in cubic graphs. Ars Math. Contemp. 4(1):79-93, 2011.

[6] F. Jaeger, T. Swart. Conjecture 1, in Combinatorics 79 (M. Deza and I. G. Rosenberg eds.), Ann. Discrete Math. 9:305, North-Holland, Amsterdam, 1980. 
[7] R. Lukotkka, E. Máčajová, J. Mazák, M. Škoviera. Small snarks with large oddness, arXiv: 1212.3641 [cs.DM].

[8] V. G. Vizing. On an estimate of the chromatic class of a p-graph. Diskret. Analiz. 3:25-30, 1964. 